\title{
Statin therapy might reduce inflammation in Crohn's disease
}

Treatment with the statin, atorvastatin, significantly reduces plasma levels of the inflammatory CXC chemokine ligand, CXCL10, in patients with Crohn's disease, report Olof Grip and Sabina Janciauskiene.

Upregulation of several inflammatory chemokines and their receptors correlates with Crohn's disease activity; Grip and Janciauskiene have previously shown that high-dose atorvastatin reduces levels of C-reactive protein, a marker of intestinal inflammation, and has clinical activity in Crohn's disease patients. Given that statins reduce the expression of chemokines and their receptors in patients with coronary heart disease, these investigators hypothesized that atorvastatin would also reduce chemokine levels in patients with Crohn's disease.

They analyzed plasma samples from 10 patients before and after treatment with atorvastatin ( $80 \mathrm{mg}$ daily for 13 weeks) for nine inflammatory chemokines and four endothelial cytokines. Only CXCL10 levels were significantly different in all treated patients, with a reduction of $34 \%$ between baseline and the end of treatment. In addition, plasma levels of CXCL10 correlated with those of C-reactive protein before and after treatment $(r=0.82)$.

When CXCL10 binds to its receptor, CXCR3, activation results in T-lymphocyte recruitment and mucosal inflammation. Thus, reducing CXCL10 levels with atorvastatin should be further investigated as a treatment for Crohn's disease.

\section{Ezzie Hutchinson}

Original article Grip, $0 . \&$ Janciauskiene, S. Atorvastatin reduces plasma levels of chemokine (CXCL10) in patients with Crohn's disease. PLoS One 4, e5263 (2009). 